Fluctuations and pinch-offs observed in viscous fingering

Moore, M. G. and Juel, A. and Burgess, J. M. and Swinney, H. L.

2002

MIMS EPrint: 2007.134

Manchester Institute for Mathematical Sciences

School of Mathematics

The University of Manchester

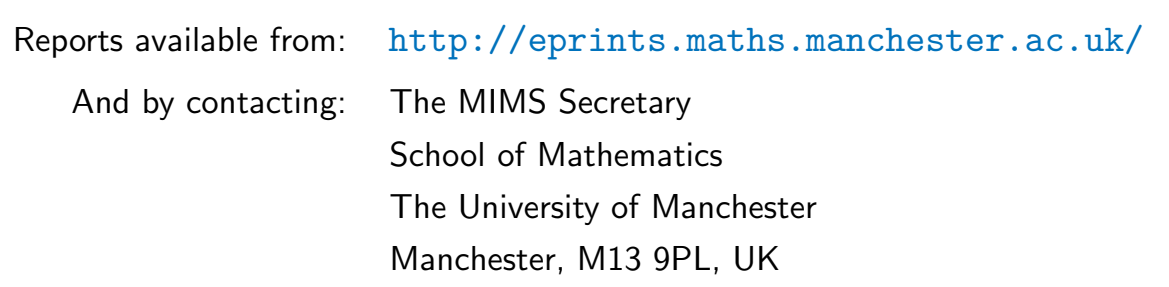

ISSN 1749-9097 


\title{
Fluctuations and Pinch-Offs Observed in Viscous Fingering
}

\author{
Mitchell G. Moore*, Anne Juel*†, John M. Burgess*, W. D. McCormick* \\ and Harry L. Swinney* \\ *Center for Nonlinear Dynamics and Department of Physics, \\ The University of Texas at Austin, Austin, Texas, 78712 \\ ${ }^{\dagger}$ Present address: Dept. of Mathematics, \\ University of Manchester, Manchester MI3 9PL, United Kingdom
}

\begin{abstract}
.
Our experiments on viscous (Saffman-Taylor) fingering in Hele-Shaw channels reveal several phenomena that were not observed in previous experiments. At low flow rates, growing fingers undergo width fluctuations that intermittently narrow the finger as they evolve. The magnitude of these fluctuations is proportional to $\mathrm{Ca}^{-0.64}$, where $\mathrm{Ca}$ is the capillary number, which is proportional to the finger velocity. This relation holds for all aspect ratios studied up to the onset of tip instabilities. At higher flow rates, finger pinch-off and reconnection events are observed. These events appear to be caused by an interaction between the actively growing finger and suppressed fingers at the back of the channel. Both the fluctuation and pinch-off phenomena are robust but not explained by current theory.
\end{abstract}

Viscous fingering occurs when a less viscous fluid displaces a more viscous fluid in a Hele-Shaw channel (a quasi-2D geometry in which the width $w$ is much greater than the channel thickness $b$ ); the interface between the fluids is unstable and forms a growing pattern of "fingers". A single finger forms at low flow rates; more complex branched patterns evolve at high flow rates. This phenomenon is the simplest example of the class of interfacial pattern forming systems which includes dendritic growth and flame propagation. Viscous fingering thus continues to receive attention for the insight it provides into these important problems $[1,2,3,4]$.

Saffman and Taylor first studied the problem in 1958 [5] by injecting air into oil in a Hele-Shaw cell. They observed the formation of a single, steadily moving finger whose width decreased monotonically to $1 / 2$ of the channel width as the finger speed was increased. Subsequent experimental [6], numerical [7], and theoretical $[8,9]$ work found that the ratio of finger width to channel width, $\lambda$ depended on a modified capillary number, $1 / B=12(w / b)^{2} \mathrm{Ca}$, which combines the aspect ratio, $w / b$, and the capillary number, $\mathrm{Ca}=\mu V / \sigma$, where $\mu$ is the dynamic viscosity of the liquid, $V$ is the velocity of the tip of the finger, and $\sigma$ is the surface tension. A transition to complex patterns of tip-splitting occurs at large $1 / B$ values $[6,10,11,12]$. Our experiments have revealed two phenomena that were not reported in prior experiments $[5,6,10,11]$ or predicted theoretically: fluctuations in the width of the evolving viscous fingers [13], and finger pinch-off events.

CP676, Experimental Chaos: $7^{\text {th }}$ Experimental Chaos Conference, edited by V. In, L. Kocarev, T. L. Carroll, B. J. Gluckman, S. Boccaletti, and J. Kurths (C) 2003 American Institute of Physics 0-7354-0145-4/03/\$20.00 


\section{EXPERIMENTAL METHODS AND DATA ANALYSIS}

We conducted experiments in a $254 \mathrm{~cm}$ long channel formed from $1.9 \mathrm{~cm}$ thick glass plates. The spacing between the glass plates was set by stainless steel strips with thicknesses $b=0.051 \mathrm{~cm}, 0.064 \mathrm{~cm}, 0.102 \mathrm{~cm}$, or $0.127 \mathrm{~cm}$; the channel width $w$ between the spacers was varied between $19.9 \mathrm{~cm}$ and $25.1 \mathrm{~cm}$. Both glass plates were supported and clamped at the sides, and the channel was illuminated from below. For aspect ratios under 150 , we used a smaller channel of length $102 \mathrm{~cm}$ and width $7.4 \mathrm{~cm}$. Interferometric measurements revealed that the root-mean-square variations in gap thickness were typically $0.6 \%$ or less in the large channel and $0.8 \%$ in the small channel. Mechanical measurements of the bending of the glass due to the imposed pressure gradient revealed that such deflections were typically $0.2 \%$ or less; the maximum deflection $(2.2 \%)$ was measured in the widest channel close to the oil reservoir at the highest flow rates. Experiments were conducted with air penetrating a Dow Corning silicone oil whose surface tension and dynamic viscosity was either $\sigma=19.6$ dyne $/ \mathrm{cm}, \mu=9.21 \mathrm{cP}$ or $\sigma=20.6$ dyne $/ \mathrm{cm}, \mu=50.8 \mathrm{cP}$ at laboratory temperature $\left(22^{\circ} \mathrm{C}\right)$. The oils wet the glass completely. We withdrew oil at a uniform rate using a syringe pump attached to a reservoir at one end of the channel; an air reservoir at atmospheric pressure was attached to the other end. The entire experiment was placed on a floating optical table to minimize vibrations and allow precise levelling of the channel.

We obtained images of up to $1200 \times 10,000$ pixels at a resolution of $0.25 \mathrm{~mm} /$ pixel using a camera and a rotating mirror. The camera captured up to 11 overlapping frames which were then concatenated, background subtracted, and corrected for perspective effects. The interfaces were then digitally traced, yielding finger width values accurate to $0.1 \%$ in the larger channel and $0.3 \%$ in the smaller channel. For each flow rate, up to four time sequences of 20-30 digital interfaces were recorded. Finger widths determined in consecutive sequences agreed within the measurement accuracy. Mean width values agreed within $0.5 \%$ for data sets repeated after channel disassembly, cleaning, and reassembly. For each experimental run, we determined the time average $\langle\lambda\rangle$ and the root-mean-square (rms) fluctuation from the mean $\delta_{\lambda}$ (as described in [13]). Each data set was analyzed for flow rates up to the point of tip splitting, beyond which the finger width $\lambda$ was no longer well defined '.

\section{FLUCTUATIONS}

Typical interface image sequences are shown for $w / b=158$ and 490 in Fig. 1. The finger width $\lambda$ (relative to the channel width $w$ ) fluctuates visibly at low flow velocities for both aspect ratios (Fig. 1 (a),(c)). In the smaller aspect ratio system the width appears to become steady as the finger velocity is increased (Fig. 1(b)), appearing exactly like the classic "half-width finger" of Saffman and Taylor. However, with sufficient resolution,

\footnotetext{
1 The first tip instabilities observed with increasing $1 / B$ are asymmetric "shouldering" modes, not actual tip splitting $[1,2,3,4,6]$; because the first instabilities are not obviously distinguishable from fluctuations, our data at high $1 / B$ include $\langle\lambda\rangle$ values averaged over such instabilities.
} 
$\mathrm{w} / \mathrm{b}=158$

(a)

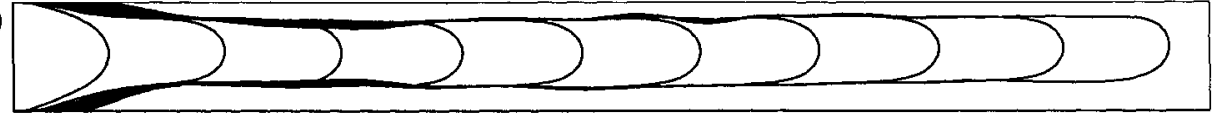

(b)

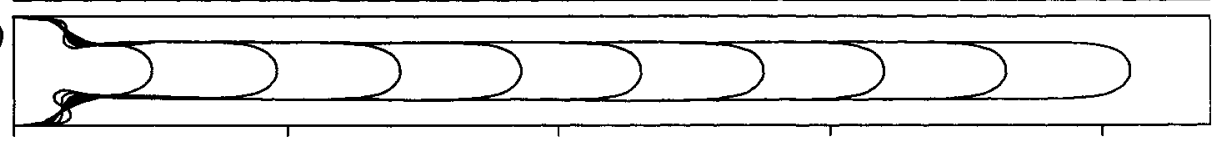

$w / b=490$

(c)

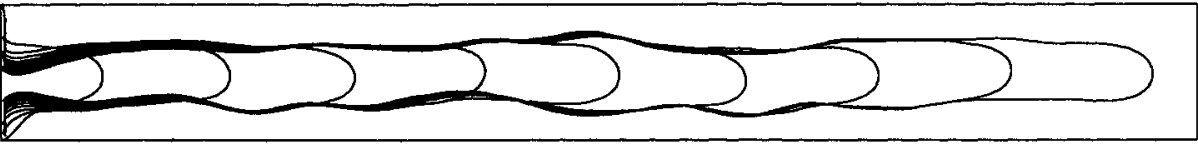

(d)

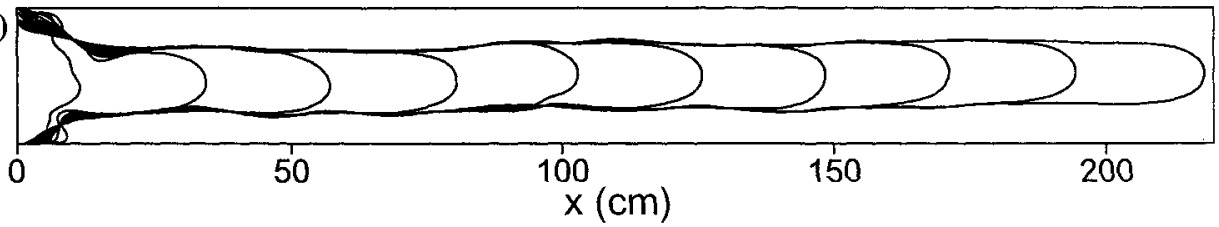

FIGURE 1. Finger images recorded at regular time intervals for different values of aspect ratio $w / b$ and modified capillary number $1 / B$. Experimental values for each run: (a) $1 / B=116$, the rms fluctuation of the finger width $\delta_{\lambda}=1.30 \times 10^{-2}$, time between successive curves $\Delta t=270$; (b) $1 / B=1757$, $\delta_{\lambda}=3.96 \times 10^{-3}, \Delta t=18 ;$ (c) $1 / B=408, \delta_{\lambda}=3.32 \times 10^{-2}, \Delta t=810 ;$ (d) $1 / B=2869, \delta_{\lambda}=1.26 \times 10^{-2}$, $\Delta t=108$.

fluctuations can still be measured for all velocities up to the onset of tip instabilities. In the higher aspect ratio system the width fluctuates visibly for all flow rates (Fig. 1(d)). We find that the rms fluctuation of the finger widths (relative to the channel width $w$ ) is described by $\delta_{\lambda}=A(\mathrm{Ca})^{\beta}$ with $A=(1.1 \pm 0.3) \times 10^{-4}$ and $\beta=-0.64 \pm 0.04$, for all the aspect ratios studied (Fig. 2).

The fluctuations in finger width are accompanied by a substantial deviation from the expected relation between finger width and velocity [13]. For low aspect ratios, our finger width measurements are in accord with previous results, but for aspect ratios $w / b \gtrsim 250$, which were not examined in previous work, we find that the mean finger width exhibits a surprising maximum as the tip velocity is decreased. However, the maximum value of the fluctuating finger width observed during finger evolution, $\lambda_{\max }$, still exhibits the classical scaling with $1 / B$ for all aspect ratios. This suggests that the fluctuations represent an intermittent narrowing of the fingers from their "ideal" width [13].

The finger width fluctuations and the peak in $\langle\lambda\rangle$ versus $1 / B$ have proven robust under many variations of experimental conditions, allowing us to rule out many possible explanations for the phenomena. We obtained identical results with differences in the oil viscosity, the pinning properties at the back of the channel, the pumping method, and the magnitude of the channel's intrinsic gap variations [13]. An instability of the film wetting layer is also unlikely, because the film is very thin at low capillary numbers, where 


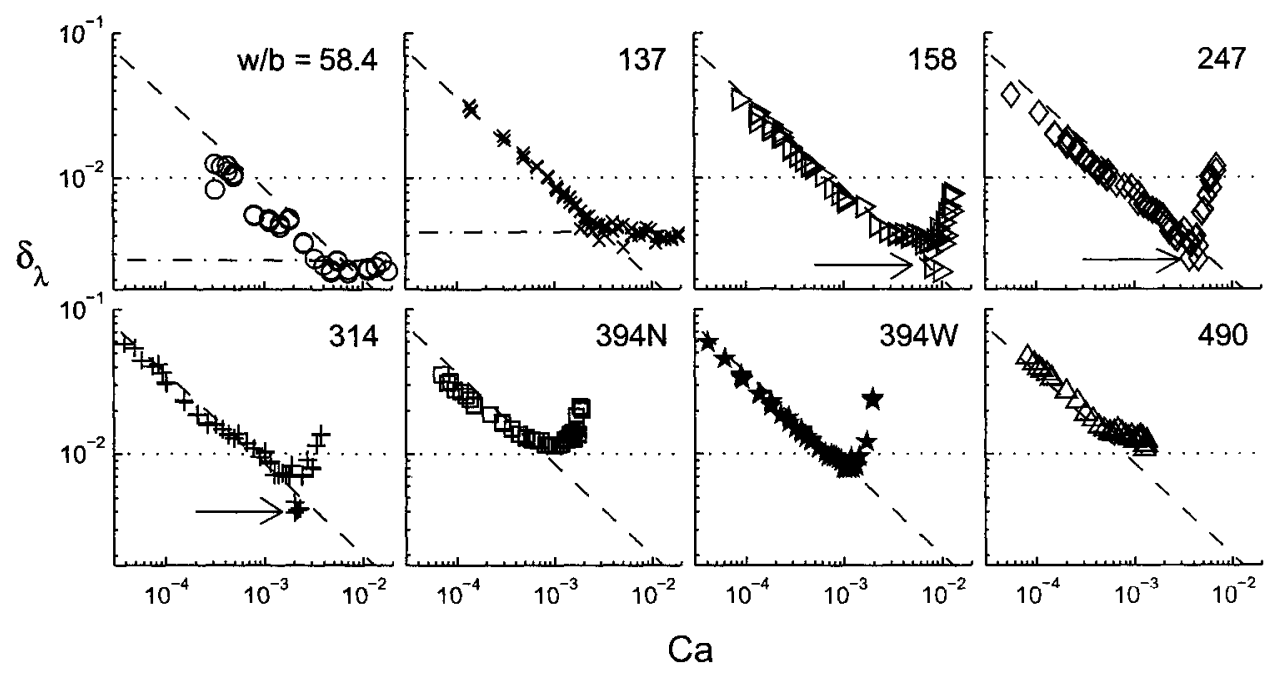

FIGURE 2. The rms fluctuation of the relative finger width $\delta_{\lambda}$ as a function of capillary number Ca, where the dashed lines describe the best fit to all of the data sets within the observed scaling region, $\delta_{\lambda}=\left(1.1 \times 10^{-4}\right) \mathrm{Ca}^{-0.64}$. (The data for $394 \mathrm{~N}$ and $394 \mathrm{~W}$ have the same aspect ratio but different widths; the channel for $394 \mathrm{~N}$ was $20.0 \mathrm{~cm}$ wide, $394 \mathrm{~W}$ was $25.0 \mathrm{~cm}$.) The first two graphs are for data taken in the smaller channel; the horizontal dash-dotted lines correspond to the limits of measurement accuracy for that channel. Fluctuations with $\delta_{\lambda} \lesssim 10^{-2}$ are not obvious visually, which may explain why previous experiments at lower aspect ratios did not observe them. An upturn in $\delta_{\lambda}$ occurs at high $\mathrm{Ca}$, signalling the onset of the secondary instabilities in the tips of the fingers. The arrows in the graphs for $w / b=158,247$, and 314 point to data from runs where pinch-off events (see fig. 3) occurred, demonstrating the delay of the onset of secondary instabilities in these cases.

the fluctuations are largest. Though the fluctuation power law we observed remained unchanged for all experimental variations, we did discover that measurements on two channels with $w / b=394$ but different values of $w$ and $b$ yielded slightly different results [13]. This difference suggests that either the finger width for a given $w / b$ and $\mathrm{Ca}$ is not unique, or perhaps a third parameter, as yet unknown, is necessary to fully describe the problem.

\section{PINCH-OFF EVENTS}

In addition to finger width fluctuations, we have observed another unexpected phenomenon: finger pinch-off events followed by reconnection to a different finger, an example of which is shown in fig. 3 . For sufficiently high flow rates, several fingers compete during the early stages until one gets ahead, suppressing the growth of the other initial fingers. However, if the initial competition stage was long, the suppressed fingers will continue to interact with the active finger. As the active finger continues to grow, it narrows at the back, and the adjacent short finger grows towards it. This process is 
(a)
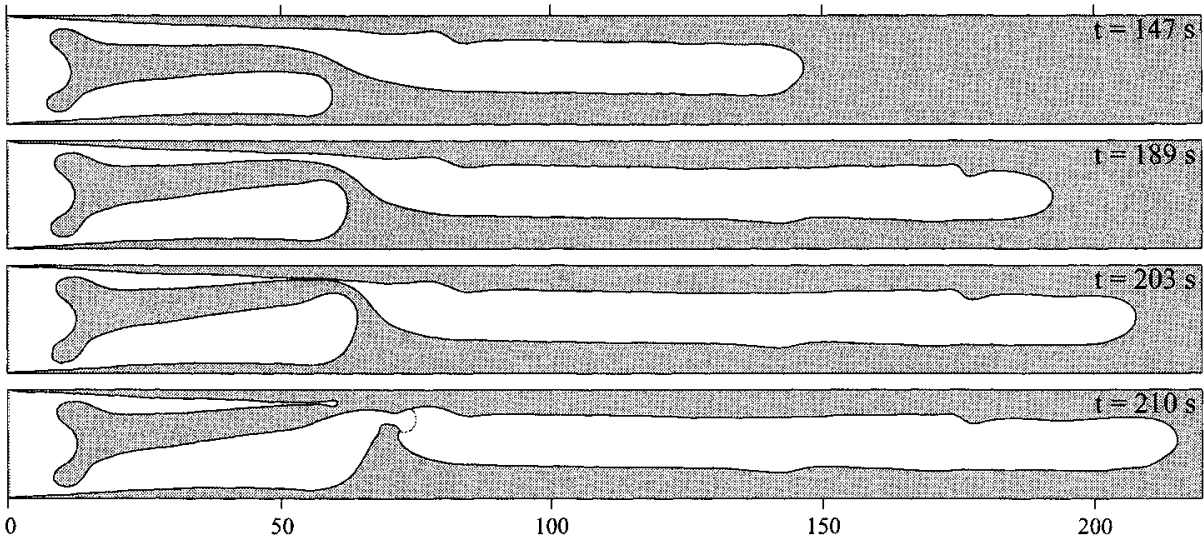

(b)
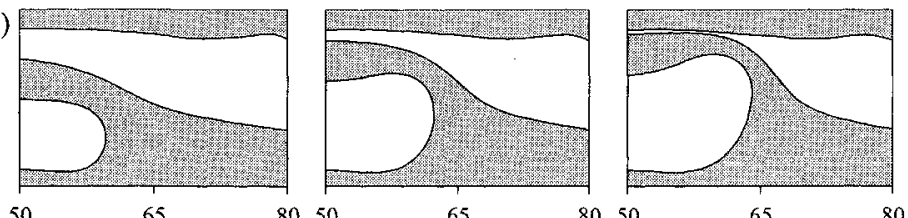

8050

65

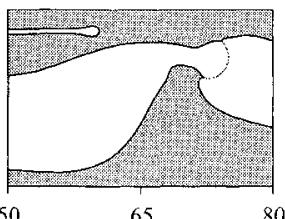

FIGURE 3. (a) A time sequence from a run where a pinch-off event occurred; the times since the beginning of the pumping are shown. The back of the growing finger narrows while the adjacent suppressed finger grows. The process accelerates as it continues; the pinch-off and subsequent reconnection happens in less than two seconds (which is faster than the time resolution we had for this data). The gray line in the last interface shows where the interface between the middle finger and the disconnected bubble finally broke; this point could be clearly seen on the image as a thick film left behind at that point. (b) A close up view of the pinch-off at the same times. (The distance scales shown are in $\mathrm{cm}$.)

very slow at first and gradually accelerates; the final pinch-off and the connection of the previously suppressed finger to the resulting bubble occurs very quickly.

The dynamics of the finger tip appear unaffected during these events, though the onset of secondary instabilities is apparently slightly suppressed. This can be seen in fig. 2; all of the data points indicated by arrows had pinch-off events, and they continue to follow the fluctuation power-law when other runs at similar flow rates without pinchoff events depart that curve, beginning the upturn that marks the onset of the secondary instabilities. At higher flow rates, pinch-offs at the back of fingers and tip instabilities both occur, apparently independently of one another.

Pinch-offs may or may not occur on a particular run at a given flow rate, since this depends on the configuration of the initial finger competition, which can be different each time. In particular, the levelling of the cells is crucial: a small tilt around the long axis will give fingers on the higher side of the cell an early advantage; the shorter dormant fingers resulting from this do not interact as quickly.

Pinch-off events are not mentioned in any of the previous literature; we believe that we observe it because our cell has an unusually high ratio of length to width, allowing 
sufficient time for evolution towards the pinch-off to occur before the finger reaches the end of the channel. Also, seemingly unimportant asymmetries across the width of the cell, like the levelling mentioned above, can disrupt the long period of finger competition and thus suppress the pinch-off events. We have observed the pinch-off phenomenon in different cells at both high and low aspect ratios. These events can occur at flow rates where the plate bending due to the imposed pressure gradient is unmeasurably small. The cause of this phenomenon is still unknown.

\section{CONCLUSIONS}

In conclusion, we have discovered fluctuations that intermittently narrow evolving single fingers; the magnitude of these fluctuations follows a power law with the capillary number for all aspect ratios studied. This is accompanied by a departure from the classic scaling of finger width versus $1 / B$ for large aspect ratios $(w / b \gtrsim 250)$. In addition, we observe finger pinch-off and reconnection events at the back of the channel; these are apparently due to an interaction between adjacent fingers that has not yet been explored. Neither the fluctuation nor the pinch-off phenomena are predicted by existing viscous fingering theories, nor have they been observed in simulations.

\section{ACKNOWLEDGMENTS}

We thank J. B. Swift for frequent guidance and advice. This work was funded by the Office of Naval Research.

\section{REFERENCES}

1. Bensimon, D., Kadanoff, L. P., Liang, S., Shraiman, B. I., and Tang, C., Reviews of Modern Physics, 58, 977-999 (1986).

2. Homsy, G. M., Annual Review of Fluid Mechanics, 19, 271-311 (1987).

3. Pelcé, P., Dynamics of Curved Fronts, Academic Press, San Diego, CA, 1988.

4. Couder, Y., "Viscous Fingering as an Archetype for Growth Patterns," in Perspectives in Fluid Dynamics, edited by G. K. Batchelor, H. K. Moffatt, and M. G. Worster, Cambridge University Press, 2000.

5. Saffman, P. G., and Taylor, S. G., Proceedings of the Royal Society, A245, 312-329 (1958).

6. Tabeling, P., Zocchi, G., and Libchaber, A., Journal of Fluid Mechanics, 177, 67-82 (1987).

7. DeGregoria, A. J., and Schwartz, L. W., Journal of Fluid Mechanics, 164, 383 (1986).

8. McLean, J. W., and Saffman, P. G., Journal of Fluid Mechanics, 102, 455469 (1981).

9. McLean, J. W., The Fingering Problem in Flow Through Porous Media, Ph.D. thesis, California Institute of Technology (1980).

10. Park, C. W., and Homsy, G. M., Physics of Fluids, 28, 1583-1585 (1985).

11. Kopf-Sill, A. R., and Homsy, G. M., Physics of Fluids, 31, 242-249 (1988).

12. Maxworthy, T., Journal of Fluid Mechanics, 177, 207-232 (1987).

13. Moore, M. G., Juel, A., Burgess, J. M., McCormick, W. D., and Swinney, H. L., Physical Review E, 65, 030601 (2002). 